\title{
Fair Resource Allocation in Wireless Networks using Queue-length-based Scheduling and Congestion Control
}

\author{
Atilla Eryilmaz and R. Srikant
}

\begin{abstract}
We consider the problem of allocating resources (time slots, frequency, power, etc.) at a base station to many competing flows, where each flow is intended for a different receiver. The channel conditions may be time-varying and different for different receivers. It is well-known that appropriately chosen queue-length based policies are throughput-optimal while other policies based on the estimation of channel statistics can be used to allocate resources fairly (such as proportional fairness) among competing users. In this paper, we show that a combination of queue-length-based scheduling at the base station and congestion control implemented either at the base station or at the end users can lead to fair resource allocation and queue-length stability.
\end{abstract}

Keywords: Throughput-optimal scheduling, Congestion control, $m$-weighted fairness, Proportional fairness, Wireless networks.

\section{INTRODUCTION}

The wireless channel is a shared medium over which many users compete for resources. Since there are many users, it is important to allocate this shared resource in a fair manner among the users. Further, since the available spectrum is limited, it is also important to efficiently use the channel. However, the time-varying nature of the wireless environment, coupled with different channel conditions for different users, poses significant challenges to accomplishing these goals. Moreover, the lack of availability of channel and arrival statistics further complicates the solution.

We will primarily restrict our attention to the downlink in cellular networks where there is a base station that allocates resources to many competing users. We assume that the packets destined for the different receivers are stored in separate queues. The scheduler at the base station is responsible for allocating resources to the different queues as a function of the current channel conditions as well as the queue-lengths. Prior work on this problem can be largely classified into two main categories:

- Throughput-optimal scheduling: Here it is assumed that the mean arrival rates of the packets into each queue lie within the capacity region (the set of sustainable arrival rates)

Research supported by a Vodafone Fellowship, DARPA Grant F30602-002-0542 and AFOSR URI F49620-01-1-0365.

An earlier version of the paper appeared in the Proceedings of Infocom 2005.

This work was completed during Atilla Eryilmaz's graduate studies at the University of Illinois at Urbana, Champaign. He is currently with the Laboratory for Information and Decision Systems at the Massachusetts Institute of Technology \{eryilmaz@mit.edu\}.R. Srikant is with the University of Illinois at Urbana, Champaign \{rsrikant@uiuc.edu\}. of the channel. However, neither the actual arrival rates nor the channel capacity region is assumed to be known. The scheduler is allowed to know the current queue-lengths and the current channel conditions. It was then shown in [38] that allocating resources to maximize a queue-length-weighted sum of the rates (which are feasible in the current time slot) is a stabilizing policy. This result was then generalized in many different directions in [3], [33], [32], [12], [4], [23], [10], [28]. Such policies are called throughput optimal since the queues are stable if the arrival rates lie within the capacity region.

- Fair Scheduling: An obvious drawback of throughputoptimal policies is that no traffic policing is enforced. For instance, if one or more sources misbehave and increase their arrival rates so that the set of arrival rates lies outside the capacity region, then the system becomes unstable. In other words, all flows will be penalized due to the behavior of a few misbehaving flows. Thus, an alternative is to provide some degree of flow isolation at least in the long term, by allocating resources in a fair manner to the various queues. A commonly-used framework for such allocation is the concept of proportional fairness [16]. It was shown in [39] that proportional fairness can be achieved in TDMA cellular networks by scheduling the user which has the largest ratio of the achievable data rate at the current instant to the average rate that it has been allocated so far. The properties of such a policy have been studied empirically in [15] and analytically in [36], [21], and a multiple antenna implementation of such an algorithm over slowly time-varying channels has been proposed in [40]. Related work on channel-state-aware scheduling in wireless networks can also be found in [1], [9], [25], [8], [26].

From an applications point of view, throughput-optimal scheduling as described above is more suitable for inelastic traffic where the sources do not adapt their transmission rate based on congestion in the network. In this case, admission control is required to ensure that the arrival rates lie within the capacity region of the network and further, in the case of wireless networks, due to the time-varying nature of the network, an appropriate scheduling algorithm is required to ensure that the network can stably serve the admitted traffic. On the other hand, fair scheduling is more suited for elastic traffic sources which can adjust their traffic rates in response to feedback from the network regarding the network conditions. Without such a rate-control mechanism, fair scheduling would either lead to under utilization (when a traffic source is not generating enough data to make use of the bandwidth allocated to it) or packet losses or large delays (when a traffic source 
is generating data at a much larger rate than the rate allocated to it by the base station).

In this paper, we are interested in allocating resources to elastic sources whose utilities are described by concave functions. Specifically, user $i$ derives a utility $U_{i}\left(a_{i}\right)$ when it transmits at rate $a_{i}$. For ease of exposition, we consider utility functions of the form

$$
U_{i}\left(a_{i}\right)=\beta_{i} \frac{a_{i}^{\left(1-\frac{1}{m}\right)}}{\left(1-\frac{1}{m}\right)}, \quad \text { for each } i \in\{1, \cdots, n\},
$$

where $m$ is a positive constant and $\beta_{i}$ is some fixed weight, which can be different for different users. Thus, we consider $m$-weighted fair resource allocation. Notice that as $m \rightarrow 1$, this allocation converges to the weighted proportionally fair allocation and as $m \rightarrow 0$, it gives the weighted max-min fair allocation. We assume that congestion information is conveyed to the sources by putting the corresponding congestion price in the ACK packets. Each source react to its congestion price by choosing its transmission rates such that its marginal utility $\left(U_{i}^{\prime}\left(a_{i}\right)\right)$ is equal to the congestion price. We take the queuelength at the base station to be the congestion price. In the Internet context, this is a special case of the dual algorithm proposed in [27], [43], [30]. In wireline networks, this interpretation of queue-length (or delay) as the congestion price naturally arises from an convex optimization perspective where the resource constraints are linear [34]. However, in wireless networks, this interpretation is not immediately obvious since the resource constraints are not necessarily linear. Despite this, we show that the dual algorithm at the sources, along with queue-length-based scheduling at the base station, can be used to approximate $m$-weighted fairness arbitrarily closely, where the approximation depends on the choice of a certain parameter used in the congestion control algorithm. Instead of the dual algorithm other algorithms such as the primal algorithm [17], [20] and the primal-dual algorithm [42], [2] can also be used. For a comprehensive description of the many algorithms that can be used to solve the resource allocation problem, see [34]. In this paper, we restrict ourselves to the dual algorithm. The problem studied in this paper has also been considered in [35]. The results in [35] are independent and contemporaneous to our work. However, the solution proposed in [35] is quite different. The solution in [35] uses a greedy source rate update rule while we use a static update rule as we will see later.

The paper is organized as follows. Section II describes the model, including the scheduling and the congestion control algorithms. In Section III, we first analyze a heuristic continuous-time, deterministic fluid model of the system and then use the intuition thus obtained to study the original stochastic system model. Generalizations and implementation considerations are discussed in Section IV. Various simulation results are presented in Section V. Finally, conclusions are drawn in Section VI.

\section{SySTEM MOdEL}

We consider a cellular network shared by $n$ flows in the downlink. Figure 1 depicts the combination of the congestion controller and the base station from the perspective of a single flow. We assume that time is slotted and denote the size of the $i^{\text {th }}$ queue at the beginning of the time slot $t$ by $x_{i}[t]$, the number of arrivals to queue $i$ in time slot $t$ by $a_{i}[t]$, and the amount of service offered to queue- $i$ in slot $t$ by $\left.\mu_{i}^{[} t\right]$. We assume that each of these parameters can only take nonnegative integer values. The evolution of the size of the $i^{t h}$ queue is given by

$$
x_{i}[t+1]=\left(x_{i}[t]+a_{i}[t]-\mu_{i}[t]\right)^{+}, \quad i=1, \cdots, n
$$

where $(y)^{+}$is equal to $y$ if $y>0$, and zero otherwise.

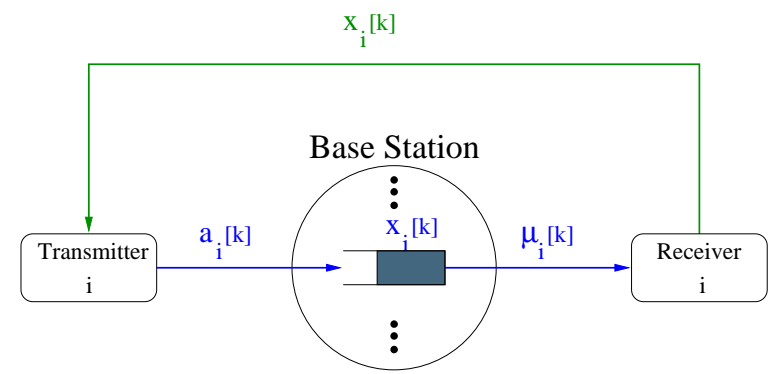

Fig. 1. A pictorial depiction of the system.

We assume that the channel between the base station and the receivers can be in one of $J$ states in a given slot. We use $s[t]$ to denote the state in time slot $t$. The channel state is assumed to be fixed within a time slot, but may vary from one slot to another, and hence captures the time-varying characteristics of a fading environment. Corresponding to each channel state, say $j$, is an achievable rate region, $\mathcal{C}_{j}$, that is defined to be the convex hull of the rate vectors, $\eta[t]:=\left(\eta_{1}[t], \cdots, \eta_{n}[t]\right)^{\prime}$, that can be offered to the queues. We assume that each $\mathcal{C}_{j}$ is a bounded region, which is a reasonable assumption since in practice, power constraints and code designs allow only finite rate levels. We use $\hat{\eta}<\infty$ to denote the upper bound on the achievable rates for all channel states. The channel state process is assumed to be independent and identically distributed in each time slot (although it is straightforward to generalize our results to allow Markovian channel state processes - see [12]), but we do not require that the statistics be known at the base station. We define the mean achievable

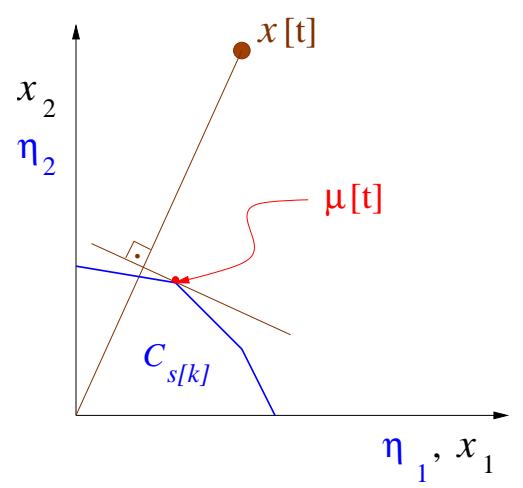

Fig. 2. Given $\mathbf{x}[t]$ and $s[t]$, the vector $\mu[t]$ is chosen on the boundary of the current achievable rate region as in this figure. 
rate region as

$$
\overline{\mathcal{C}}:=\left\{\eta: \eta=\sum_{j=1}^{J} \pi_{j}^{c h} \eta^{(j)}, \eta^{(j)} \in \mathcal{C}_{j}\right\},
$$

where $\pi_{j}^{c h}$ stands for the stationary probability of the channel state process being in state $j$. The scheduler implemented at the base station is described as follows.

QUEUE-LENGTH-BASED SCHEDULER: In time slot $t$, given the current queue-length vector, $\mathbf{x}[t]:=\left(x_{1}[t], \cdots, x_{n}[t]\right)^{\prime}$, and the current channel state, $s[t]$, the scheduler chooses a service rate vector $\mu[t]:=\left(\mu_{1}[t], \cdots, \mu_{n}[t]\right)^{\prime} \in \mathcal{C}_{s[t]}$ that satisfies

$$
\mu[t] \in \arg \max _{\eta \in \mathcal{C}_{s[t]}} \sum_{i=1}^{n} x_{i}[t] \eta_{i} .
$$

A geometric interpretation of the above policy for two users is given in Figure 2, where the queue-length state space and the achievable service rate region are superimposed on each other. We observe that the allocated service rates always lies on the boundary of the current achievable rate region, and that if the rate vector $\mu[t]$ satisfies (2) for some $\mathbf{x}[t]$, then it satisfies the same condition for all $\xi \mathbf{x}[t]$, for all $\xi>0$. As mentioned earlier, this policy is known to be throughputoptimal for inelastic flows. We note that it is possible that there are more than one $\mu[t]$ that satisfies (2) in a given time slot. In such a case, the choice of $\mu[t]$ must be made so that the chosen vector is in fact achievable in that slot. Such a choice is possible since within every optimal set, there must exist at least one rate vector that is achievable because the extreme points of $\mathcal{C}_{j}$ is achievable for each $j$.

The packet arrival rate into the queue is assumed to be congestion controlled according to the dual controller [27], [43]. We note that the dual controller is derived for the Internet applications where all users sharing a common resource can receive the same treatment from the router. However, here the situation is fundamentally different due the fact that the channel conditions for the different users can be different. One of the contributions of this paper is show that, somewhat surprisingly, the dual controller still leads to fair resource allocation provided that the base station uses the scheduler described above. Such a scheduling mechanism is not necessary in the Internet, but is crucial in the wireless network context studied here. We now describe the congestion controller.

Congestion Controller: For the $i^{\text {th }}$ flow, given its current buffer occupancy $x_{i}[t]$, the data generation rate in slot $t$, equal to $a_{i}[t]$ in our notation, is a random variable satisfying the following:

$$
\begin{gathered}
E\left[a_{i}[t] \mid x_{i}[t]\right]=\min \left\{\frac{\alpha_{i} K}{\left(x_{i}[t]\right)^{m}}, M\right\} \\
E\left[a_{i}^{2}[t] \mid x_{i}[t]\right] \leq A<\infty, \quad \forall x_{i}[t]
\end{gathered}
$$

where $m,\left\{\alpha_{i}\right\}, A$ and $M>2 \hat{\eta}$ are positive constants ${ }^{1}$. We also assume $a_{i}[t]$ is independent across time slots for each $i$. (Again, it is straightforward to generalize this assumption to allow dependence in the arrivals across time slots [13].)

\footnotetext{
${ }^{1}$ To relate to (1), the first term in the minimization of (3) is equal to $U_{i}^{\prime-1}\left(x_{i}[t]\right)$ when we define $K \alpha_{i}:=\beta_{i}^{m}$.
}

We have allowed $a_{i}[t]$ to be a random variable to allow for various sources of randomness in the actual implementation (e.g., window-based implementations of congestion control) that are not precisely modeled here. Further, we have used $M>2 \hat{\eta}$ to ensure that the arrival rate is bounded when $x_{i}[t]$ is close to zero. It is important that $M$ is large enough to ensure that any arrival rate within the mean achievable rate region is achievable by the congestion controller. We note that the results of this work will continue to hold even if $M$ is taken to be a function of $K$ that is lower bounded by $2 \hat{\eta}$ for large enough $K$. However, such a dependency is unnecessary and would cause impracticalities in the implementation. Observe the well-known basic characteristics of a congestion controller in the above mechanism: the higher the congestion level, which is indicated by an increased level of buffer occupancy, the lower the data generation rate.

Notice that we have introduced a constant $K$ in the congestion control algorithm. This corresponds to assuming that the utility function of user $i$ is $\left(K \alpha_{i}\right)^{\frac{1}{m}} \frac{a_{i}^{\left(1-\frac{1}{m}\right)}}{\left(1-\frac{1}{m}\right)}$. Since $K$ is the same for all users, this will not affect the relative resource allocation among the users. However, this constant plays a crucial role in determining how well we approximate weighted $m$-fair resource allocation in a wireless network. Indeed, we will argue that weighted $m$-fair allocation is closely approximated for large $K$. Intuitively, as $K$ increases the congestion controller reacts to the same queue-length levels more aggressively, i.e., generates more data. Such a behavior results in larger equilibrium queue-length levels and hence delay, but also in fair division of the system resources, as we will argue in the rest of the paper. In order to indicate the dependence on $K$, we will use $(K)$ as a superscript for the queue-length and service rate parameters that vary with $K$.

\section{Characterizing the System Performance}

In this section, we will analyze the system described in the previous section. To accomplish this, we will start with a heuristic continuous-time fluid model and understand its behavior. Later, we will use these observations in the analysis of the original model and show that the original model behaves like the fluid model for large $K$.

\section{A. Continuous-time Fluid Model}

In the fluid-model, we assume that the channel state process is not random, but constant at its mean level. In other words, the achievable rate region is fixed at $\overline{\mathcal{C}}$. Also, the arrival rate is no longer a random variable, but is taken to be equal to its mean, i.e., $a_{i}^{(K)}(t)=\min \left\{\frac{\alpha_{i} K}{\left(x_{i}^{(K)}(t)\right)^{m}}, M\right\}$. Here, $(t)$, instead of $[t]$, is used to signify that time is a continuous variable. Then, the evolution of the $i^{\text {th }}$ queuelength is described by

$$
\dot{x}_{i}^{(K)}(t)=\left(\min \left\{\frac{\alpha_{i} K}{\left(x_{i}^{(K)}(t)\right)^{m}}, M\right\}-\bar{\mu}_{i}^{(K)}(t)\right)_{x_{i}^{(K)}(t)}^{+},
$$


where $\bar{\mu}^{(K)}(t) \in \arg \max _{\eta \in \overline{\mathcal{C}}} \sum_{i=1}^{n} x_{i}^{(K)}(t) \eta_{i}$ and $(y)_{x}^{+}$is equal to $y$ when $x>0$ and is equal to $\max (y, 0)$ when $x \leq 0$. In this formulation, the queue-length state space is assumed to be continuous. We now identify the set of service rates and queuelengths such that if the these queue-lengths are chosen as the initial state and at each time instant, resources are allocated to achieve these service rates, then the queue-lengths will remain at the initial conditions forever.

Definition 1 (Invariant pair): The pair $\left(\stackrel{\star}{\mathbf{x}}^{(K)}, \stackrel{\star}{\mu}(K)\right)$ forms an invariant pair (more precisely, $\stackrel{\star}{\mu}^{(K)}$ is an invariant service rate vector and $\stackrel{\mathrm{x}}{(K)}^{(1)}$ an invariant queue-length vector) if they satisfy both of the following conditions:

$$
\begin{aligned}
& \left(D_{1}\right) \quad \stackrel{\star}{\mu}^{(K)} \in \arg \max _{\eta \in \mathcal{C}} \sum_{i=1}^{n} \stackrel{\star}{x}_{i}^{(K)} \eta_{i} \\
& \left(D_{2}\right) \quad \stackrel{\star}{x}_{i}^{(K)}=\left(\frac{\alpha_{i} K}{\stackrel{\star}{\mu}(K)_{i}^{(K)}}\right)^{1 / m} \quad \forall i \in\{1, \cdots, n\} .
\end{aligned}
$$

Notice that, if at any time $t^{\prime}$ we have $\mathbf{x}^{(K)}\left(t^{\prime}\right)=\stackrel{\star}{\mathbf{x}}^{(K)}$ and $\mu^{(K)}\left(t^{\prime}\right)=\stackrel{\star}{\mu}^{(K)}$, then, due to $\left(D_{1}\right)$ and $\left(D_{2}\right), \dot{x}^{(K)}(t)$ given by (4) will be zero for all $t \geq t^{\prime}$. We now show that the invariant pair exists and is unique.

Proposition 1: [Existence and Uniqueness of $\left(\stackrel{\star \star}{\mathbf{x}}^{(K)}, \stackrel{\star}{\mu}^{(K)}\right)$ ] For each $K$, an invariant pair of rates and queue-lengths exists and is unique. Moreover, the unique invariant rate vector $\stackrel{\star}{\mu}^{(K)}$ is independent of $K$.

Proof: Note that the conditions on $\stackrel{\star}{\mu}^{(K)}$ given by $\left(D_{1}\right)$ and $\left(D_{2}\right)$ can be concisely written as

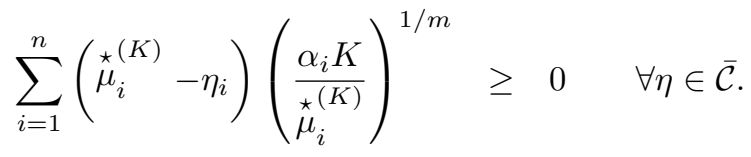

This is simply the condition for $\stackrel{\star}{\mu}^{(K)}$ to be an optimal solution to the following problem of maximizing a concave function over a convex set [6]:

$$
\stackrel{\star}{\mu}^{(K)} \in \arg \max _{\eta \in \overline{\mathcal{C}}} \sum_{i=1}^{n} \frac{\left(K \alpha_{i}\right)^{\frac{1}{m}}}{\left(1-\frac{1}{m}\right)} \eta_{i}^{\left(1-\frac{1}{m}\right)} .
$$

Since $\overline{\mathcal{C}}$ is a bounded set, clearly a solution exists to the above optimization problem. Further, since the objective is strictly concave the solution is unique. Finally, notice that $K$, being a constant factor, does not influence the optimizer of (5), hence $\stackrel{\star}{\mu}^{(K)}$ is the same for all $K$.

The above proof shows that the invariant point is simply the set of rates and queue-lengths achieved under fair resource allocation, which is our goal. Since $\stackrel{\star}{\mu}^{(K)}$ does not depend on $K$ due to Proposition 1, we will omit the superscript henceforth. Next, we are interested in showing that the queuelengths described by the invariant point is attractive, i.e., all trajectories eventually converge to it.
Proposition 2: For any fixed $K$, starting from any initial queue-length vector, $\mathbf{x}^{(K)}(0)$, the queue-length vector $\mathbf{x}^{(K)}(t)$ eventually reaches $\stackrel{\star}{\mathbf{x}}^{(K)}$ as $t \rightarrow \infty$.

Proof: We will omit the superscript $(K)$ throughout the proof for ease of exposition. Consider the Lyapunov function $W(\mathbf{x})=\frac{1}{2} \sum_{i=1}^{n}\left(x_{i}-\stackrel{\star}{x}_{i}\right)^{2}$. Next, we study the time derivative of this Lyapunov function at time $t$.

$$
\begin{aligned}
\dot{W}(\mathbf{x}(t))= & \sum_{i=1}^{n}\left(x_{i}(t)-\stackrel{\star}{x}_{i}\right) \dot{x}_{i}(t) \\
= & \sum_{i=1}^{n}\left[\left(x_{i}(t)-\stackrel{\star}{x}_{i}\right)\right. \\
& \left.\left(\min \left\{\frac{\alpha_{i} K}{\left(x_{i}(t)\right)^{m}}, M\right\}-\bar{\mu}_{i}(t)+u_{i}(t)\right)\right],
\end{aligned}
$$

where $u_{i}(t)$ is a non-negative quantity which denotes the wasted service given to queue $i$ at time $t$. Note that $u_{i}(t)=0$ whenever $x_{i}(t)>0$. Thus, it is easy to see that

$$
\begin{aligned}
\dot{W}(\mathbf{x}(t)) & \leq \sum_{i=1}^{n}\left(x_{i}(t)-\stackrel{\star}{x}_{i}\right)\left(\min \left\{\frac{\alpha_{i} K}{\left(x_{i}(t)\right)^{m}}, M\right\}-\bar{\mu}_{i}(t)\right) \\
= & \sum_{i=1}^{n}\left(x_{i}(t)-\stackrel{\star}{x}_{i}\right)\left(\min \left\{\frac{\alpha_{i} K}{\left(x_{i}(t)\right)^{m}}, M\right\}-\stackrel{\star}{\mu}_{i}\right) \\
& +\sum_{i=1}^{n}\left(x_{i}(t)-\stackrel{\star}{x}_{i}\right)\left(\stackrel{\star}{\mu}_{i}-\bar{\mu}_{i}(t)\right)
\end{aligned}
$$

where the last step follows from adding and subtracting $\stackrel{\star}{\mu}$ to each term in the summation. Consider (6): if $x_{i}(t)>\stackrel{\star}{x}_{i}$ , then $\min \left\{\frac{\alpha_{i} K}{\left(x_{i}(t)\right)^{m}}, M\right\}-\stackrel{\star}{\mu}_{i}<0$; and if $x_{i}(t)<\stackrel{\star}{x}_{i}$, then $\min \left\{\frac{\alpha_{i} K}{\left(x_{i}(t)\right)^{m}}, M\right\}-\stackrel{\star}{\mu}_{i}>0$. Therefore, unless $\stackrel{\star}{\mathbf{x}}_{i}=\mathbf{x}_{i}(t)$, the expression (6) is negative and when $\stackrel{\star}{x}_{i}=\mathbf{x}_{i}(t)$, the expression (6) is zero.

Next we consider (7). We show that this expression is negative unless $\stackrel{\star}{\mathbf{x}}=\mathbf{x}(t)$. First we note the following two inequalities which follow from the definition of the invariant point and our scheduling policy, which is the solution to the optimization problem (2).

$$
\begin{aligned}
\sum_{i=1}^{n} \stackrel{\star}{x_{i}} \stackrel{\star}{\mu}_{i} & \geq \sum_{i=1}^{n} \stackrel{\star}{x}_{i} \bar{\mu}_{i}(t) \\
\sum_{i=1}^{n} x_{i}(t) \bar{\mu}_{i}(t) & \geq \sum_{i=1}^{n} x_{i}(t) \stackrel{\star}{\mu}{ }_{i}
\end{aligned}
$$

Adding both sides of the inequalities (8) and (9), and rearranging the terms yields

$$
\sum_{i=1}^{n}\left(x_{i}(t)-\stackrel{\star}{x}_{i}\right)\left(\stackrel{\star}{\mu}_{i}-\bar{\mu}_{i}(t)\right) \leq 0
$$

Combining this result with our earlier observation regarding (6) leads to

$$
\begin{aligned}
& \dot{W}(\mathbf{x}(t))<0, \quad \text { if } \mathbf{x}(t) \neq \stackrel{\star}{\mathbf{x}}, \text { and } \\
& \dot{W}(\mathbf{x}(t))=0, \quad \text { if } \mathbf{x}(t)=\stackrel{\star}{\mathbf{x}} .
\end{aligned}
$$


The result follows from Lyapunov's global stability theorem [18].

\section{B. Original System Model}

In this section, we return to our original system model, where the arrivals and departures are integer-valued and random. We observe that the queue-length state vector $\mathbf{x}^{(K)}[t]$ evolves according to a discrete-time, discrete-space Markov chain. We first show that this Markov chain is stable, i.e., positive recurrent. The fact that the Markov chain is stable is not surprising since by our choice of congestion control, we have ensured that the mean arrival rate into a queue reduces when the queue-length is large. However, the Lyapunov function used to establish stability can be used to obtain a useful upper bound on the Euclidean distance between the invariant queue-length vector $\stackrel{\star}{\mathbf{x}}^{(K)}$ and $\mathbf{x}^{(K)}[t]$ for large $K$ and large $t$. This upper bound is then used to establish the properties of our joint congestion control-scheduling algorithm.

In the following theorem, we show that the Lyapunov function $W(\cdot)$ satisfies the Foster-Lyapunov criterion [5, Proposition I.5.3], which is sufficient to establish the positive recurrence of the Markov chain $\mathbf{x}^{(K)}$. We repeat this criterion for future reference.

Proposition 3 (Foster-Lyapunov Criterion, [5]): Suppose the chain is irreducible and let $\mathcal{S}$ be a finite subset of the state space $E$. Then, the chain is positive recurrent if for some $h: E \rightarrow \mathbb{R}$ and some $\epsilon>0$, we have $\inf _{\mathbf{x}} h(\mathbf{x})>-\infty$ and

$$
\begin{aligned}
& E[h(\mathbf{x}[t+1]) \mid \mathbf{x}[t]=\mathbf{x}] \leq B, \quad \mathbf{x} \in \mathcal{D}, \\
& E[h(\mathbf{x}[t+1]) \mid \mathbf{x}[t]=\mathbf{x}] \quad<h(\mathbf{x})-\epsilon, \quad \mathbf{x} \in E \backslash \mathcal{D}
\end{aligned}
$$

where $B$ is some finite constant.

$\diamond$

Theorem 1 (Positive Recurrence): Under the congestion control mechanism satisfying (3), and the queue-length-based scheduler satisfying (2), the Markov chain $\mathbf{x}^{(K)}[t]$ is positive recurrent.

Proof: Since $K$ is fixed, we omit the superscript $(K)$ in the proof. It is easy to see that the Markov chain has a countable state space and is irreducible and aperiodic. Consider the Lyapunov function $W(\mathbf{x})=\sum_{i=1}^{n} \frac{\left(x_{i}-\stackrel{\star}{x}_{i}\right)^{2}}{2}$. It is shown in the appendix that there exist positive constants $\delta, \zeta$ and $c$ such that

$$
\begin{aligned}
E\left[\Delta W_{t}(\mathbf{x})\right] & :=E[W(\mathbf{x}[t+1])-W(\mathbf{x}[t]) \mid \mathbf{x}[t]=\mathbf{x}] \\
& \leq-\frac{\delta}{(K)^{\frac{1}{2 m}}}\|\mathbf{x}-\stackrel{\star}{\mathbf{x}}\| \mathcal{I}_{\mathbf{x} \in \mathcal{D}^{c}}+\zeta \mathcal{I}_{\mathbf{x} \in \mathcal{D}},(10)
\end{aligned}
$$

where $\mathcal{D}:=\left\{\mathbf{y}:\|\mathbf{y}-\stackrel{\star}{\mathbf{x}}\|<c(K)^{\frac{1}{2 m}}\right\}$ and $\mathcal{D}^{c}$ is the complement of $\mathcal{D}$ in $\mathbb{Z}_{+}^{n}$. Thus, we have a Lyapunov function that has negative conditional mean drift for all queue-length vectors that have sufficiently large magnitude. The positive recurrence of the Markov Chain follows from Proposition 3 when we substitute $h(\cdot)=W(\cdot) ; E=\mathbb{Z}_{+}^{n} ; \mathcal{S}=\mathcal{D} ; B=\zeta$; and $\epsilon=\frac{\delta}{(K)^{\frac{1}{2 m}}}\|\mathbf{x}-\stackrel{\star}{\mathbf{x}}\|$.
Inequality (10) is stronger than what is necessary to prove the stability of the Markov chain. However, it is useful in establishing the following theorem which characterizes the mean distance between $\mathbf{x}^{(K)}[t]$ for large $t$ and the invariant queue-length vector $\stackrel{\star}{\mathbf{x}}^{(K)}$.

Theorem 2 (Mean Distance Bound): There exists a positive constant $\bar{c}$, that depends on the mean achievable rate region, the algorithm parameters $\left\{\alpha_{i}, \gamma_{i}\right\}$, and the moments of the channel and arrival processes, such that

$$
E\left[\left\|{\underset{\mathbf{x}}{(K)}}^{\left(K \mathbf{x}^{(K)}\right.}\right\|\right] \leq \bar{c} K^{1 /(2 m)}, \quad \text { for large } K,
$$

where $\stackrel{\infty}{\mathbf{x}}^{(K)}$ is a notation used to denote the state of the Markov chain in steady-state and $\|\cdot\|$ denotes the Euclidean distance in the $\Re^{n}$.

Proof: Here also, we omit the superscript $(K)$ for ease of exposition. We use (10) and the idea behind the proof of Pakes' lemma [31], [7] to prove the theorem. We consider the following $T$-step mean drift. For any $\mathbf{y} \geq \mathbf{0}$,

$E[W(\mathbf{x}[T]) \mid \mathbf{x}[0]=\mathbf{y}]-W(\mathbf{y})$

$$
\begin{aligned}
& =\sum_{t=0}^{T-1} E[W(\mathbf{x}[t+1])-W(\mathbf{x}[t]) \mid \mathbf{x}[0]=\mathbf{y}] \\
& =\sum_{t=0}^{T-1} \sum_{\mathbf{x} \in \mathcal{Z}_{+}^{n}}[P(\mathbf{x}[t]=\mathbf{x} \mid \mathbf{x}[0]=\mathbf{y}) \\
& \leq \quad \sum_{\mathbf{x} \in \mathcal{D}} \sum_{t=0}^{T-1} P(\mathbf{x}[t]=\mathbf{x} \mid \mathbf{x}[0]=\mathbf{y}) \\
& \quad-\frac{\delta}{(K)^{\frac{1}{2 m}}} \sum_{\mathbf{x} \in \mathcal{D}^{c}}\left\|\mathbf{x}-\mathbf{x}_{\mathbf{x}}\right\| \sum_{t=0}^{T-1} P(\mathbf{x}[t]=\mathbf{x} \mid \mathbf{x}[0]=\mathbf{y}),
\end{aligned}
$$

where $\mathcal{Z}_{+}^{n}$ denotes the set of all non-negative $n$ dimensional integer valued vectors. In the above derivation, (12) follows from the fact that $\mathbf{x}[t]$ is a Markov chain, and we have used (10) to get the inequality in (13). We note that for any $\mathbf{x} \in \mathcal{Z}_{+}^{n}$,

$$
\lim _{T \rightarrow \infty} \frac{1}{T} \sum_{t=0}^{T-1} P(\mathbf{x}[t-1]=\mathbf{x} \mid \mathbf{x}[0]=\mathbf{y})=\pi_{\mathbf{x}}^{\infty},
$$

for all $\mathbf{y}$, where $\pi^{\infty}$ denotes the stationary distribution of the Markov chain $\mathbf{x}[t]$. Next, we move $W(\mathbf{y})$ to the other side of the inequality in (13), divide both sides by $T$, and let $T$ go to infinity. This operation yields

$$
0 \leq \zeta \sum_{\mathbf{x} \in \mathcal{D}} \pi_{\mathbf{x}}^{\infty}-\frac{\delta}{(K)^{\frac{1}{2 m}}} \sum_{\mathbf{x} \in \mathcal{D}^{c}}\|\mathbf{x}-\stackrel{\star}{\mathbf{x}}\| \pi_{\mathbf{x}}^{\infty} .
$$

Re-arranging the terms and with minor manipulations, this inequality can be written as

$$
\begin{aligned}
\frac{\delta}{(K)^{\frac{1}{2 m}}} \sum_{\mathbf{x} \in \mathcal{Z}_{+}^{n}}\|\mathbf{x}-\stackrel{\star}{\mathbf{x}}\| \pi_{\mathbf{x}}^{\infty} \\
\leq \sum_{\mathbf{x} \in \mathcal{D}}\left(\zeta+\frac{\delta}{(K)^{\frac{1}{2 m}}}\|\mathbf{x}-\stackrel{\star}{\mathbf{x}}\|\right) \pi_{\mathbf{x}}^{\infty} \\
\leq(\zeta+\delta c) \sum_{\mathbf{x} \in \mathcal{D}} \pi_{\mathbf{x}}^{\infty} \leq(\zeta+\delta c)
\end{aligned}
$$


where the second inequality follows from the definition of $\mathcal{D}$. Here, the left-hand-side is nothing but $\frac{\delta E[\|\stackrel{\infty}{\mathbf{x}}-\stackrel{\star}{\mathbf{x}}\|]}{(K)^{\frac{1}{2 m}}}$. So we multiply both sides with $\frac{(K)^{\frac{1}{2 m}}}{\delta}$ to get

$$
E[\|\stackrel{\infty}{\mathbf{x}}-\stackrel{\star}{\mathbf{x}}\|] \leq\left(c+\frac{\zeta}{\delta}\right)(K)^{\frac{1}{2 m}}
$$

which completes the proof for $\bar{c}:=\left(c+\frac{\zeta}{\delta}\right)$.

In Theorem 2, we proved that the mean distance between the queue-length vector and the invariant queue-length vector cannot increase at a rate larger than $K^{1 / 2 m}$. Next, recalling the relationship $\stackrel{\star(K)}{x_{i}}=\left(\frac{\alpha_{i} K}{\hat{\leftarrow}_{i}}\right)^{1 / m}$ for each $i \in\{1, \cdots, n\}$, we can state the following theorem about the rate vectors.

Theorem 3: The mean of the stationary rate vector converges to $\stackrel{\star}{\mu}$ as $K$ increases, i.e.

$$
\left|\stackrel{\infty}{\mu}_{i}^{(K)}-\stackrel{\star}{\mu}_{i}\right| \leq \bar{c} B(K)^{-1 / 2 m}, \quad \text { for all } i \in\{1, \cdots, n\},
$$

where $B$ is a finite positive constant and $\stackrel{\infty}{\mu}^{(K)}$ represents the mean service rate provided by our algorithm under the stationary distribution of the Markov Chain. More precisely, $\stackrel{\infty}{\mu}^{(K)}$ is defined by $\stackrel{\infty}{\mu}_{i}^{(K)}=E\left[\min \left\{\frac{\alpha_{i} K}{\left(\begin{array}{c}\infty \\ x_{i}(K)\end{array}\right)^{m}}, M\right\}\right]$ for all $i \in\{1, \cdots, n\}$.

Proof: For ease of presentation, let us define $U_{i}(z):=$ $\frac{\alpha_{i} z^{(1-m)}}{(1-m)}$ for each $i$. Notice that $U_{i}(\cdot)$ is a strictly concave function that satisfies

$$
0 \leq-\frac{1}{U_{i}^{\prime \prime}(z)} \leq B<\infty, \quad \text { for all } z \in[0, M]
$$

where $B$ may depend on $m$. Also, notice that the relationship between $\mathbf{x}^{(K)}$ and $\mu^{(K)}$ is given by

$$
\begin{aligned}
E\left[\mu_{i}^{(K)}[t] \mid x_{i}^{(K)}[t]\right] & =\min \left\{U_{i}^{\prime-1}\left(\frac{x_{i}^{(K)}[t]}{K^{1 / m}}\right), M\right\}, \\
\stackrel{\mu}{\mu}_{i} & =U_{i}^{\prime-1}\left(\frac{\stackrel{\star}{x}_{i}^{(K)}}{K^{1 / m}}\right),
\end{aligned}
$$

for each $i \in\{1, \cdots, n\}$. If we divide both sides of the expression (11) by $K^{1 / m}$, then we can write: for each $i \in$ $\{1, \cdots, n\}$,

$$
\begin{aligned}
E\left[\left|\frac{\stackrel{\infty}{i}_{i}^{(K)}}{K^{1 / m}}-\frac{\star_{i}^{(K)}}{K^{1 / m}}\right|\right] & \leq \bar{c} K^{-1 /(2 m)} \\
& \rightarrow 0, \quad \text { as } K \rightarrow \infty
\end{aligned}
$$

We can write

$$
\begin{aligned}
& \left|\stackrel{\infty}{\mu}_{i}^{(K)}-\stackrel{\star}{\mu}_{i}\right| \\
& =\left|E\left[\min \left\{U_{i}^{\prime-1}\left(\frac{x_{i}^{(K)}}{K^{1 / m}}\right), M\right\}\right]-U_{i}^{\prime-1}\left(\frac{\stackrel{x}{x}_{i}^{(K)}}{K^{1 / m}}\right)\right| \\
& \leq E\left[\left|\min \left\{U_{i}^{\prime-1}\left(\frac{x_{i}^{(K)}}{K^{1 / m}}\right), M\right\}-U_{i}^{\prime-1}\left(\frac{\stackrel{\star}{x}_{i}^{(K)}}{K^{1 / m}}\right)\right|\right] \\
& =E\left[\left|\frac{1}{U_{f}^{\prime \prime}(\tilde{x})}\right|\left|\frac{\stackrel{\infty}{x}_{i}^{(K)}}{K^{1 / m}}-\frac{\stackrel{\star}{x}_{i}^{(K)}}{K^{1 / m}}\right|\right] \quad \forall i \in\{1, \cdots, n\}
\end{aligned}
$$

for some $\tilde{x} \in[0, M]$ due to Taylor's expansion. Thus, we can invoke the fact (14) on the utility functions to claim that

$$
\begin{aligned}
\left|\stackrel{\infty}{\mu}_{i}^{(K)}-\stackrel{\star}{\mu_{i}}\right| & \leq B E\left[\left|\frac{\stackrel{\infty}{x}_{i}^{(K)}}{K^{1 / m}}-\frac{\stackrel{\star}{x_{i}^{(K)}}}{K^{1 / m}}\right|\right] \\
& \leq \bar{c} B K^{-1 /(2 m)}, \quad \forall i \in\{1, \cdots, n\}
\end{aligned}
$$

where the second inequality follows from (15).

This results proves that the steady state distribution converges to the fair allocation as $K$ tends to infinity. In the next statement, we use the positive recurrence of the Markov Chain $\left\{\mathbf{x}^{(K)}[t]\right\}$ to prove convergence to $\stackrel{\infty}{\mu}^{(K)}$.

Theorem 4: For any fixed $K$ and starting from any initial queue-length vector, $\mathbf{x}[0]$, the scheduling-congestion-control algorithm satisfies, for each $i \in\{1, \cdots, n\}$,

$\lim _{T \rightarrow \infty} \frac{1}{T} \sum_{t=0}^{T-1} \min \left\{\frac{\alpha_{i} K}{\left(x_{i}^{(K)}[t]\right)^{m}}, M\right\}=\stackrel{\infty}{\mu}_{i}^{(K)}$, almost surely.

Proof: We omit the superscript $(K)$ for ease of exposition. Since the Markov Chain is positive recurrent, for any bounded function $f(x)$, we have [29]

$$
\lim _{T \rightarrow \infty} \frac{1}{T} \sum_{t=0}^{T-1} f(x[t])=\bar{f}, \quad \text { almost surely, }
$$

where $\bar{f}$ denotes the mean of $f(x)$ over the steady-state distribution of the Markov Chain. Since $\min \left\{\frac{\alpha_{i} K}{\left(x_{i}[t]\right)^{m}}, M\right\}$ is a bounded function, this result applies to the Markov Chain $\{\mathbf{x}[t]\}$.

Theorems 3 and 4 characterize the convergence of the user rates to the solution of the utility optimization problem. Theorem 4 states that the empirical average of the user rates converges to certain values determined by the steady-state queue-length distribution. Theorem 3 states that these values are close to the solution of the optimization problem for large $\mathrm{K}$.

Recalling Theorem 2 we can expect the mean of $\underset{\mathbf{x}}{\infty^{(K)}}$ to be very close to $\mathbf{x}^{(K)}$. In fact, using a Lyapunov function of the form $L(\mathbf{x})=\frac{1}{2} \sum_{i=1}^{n} x_{i}^{2}$, and using arguments very similar to the ones used in the proof of Theorem 2, we can prove that for large enough $K, E\left[\begin{array}{l}\mathbf{x}^{(K)} \\ \mathbf{x}\end{array}=\Theta\left(\stackrel{\star}{\mathbf{x}}^{(K)}\right) \cdot{ }^{2}\right.$ Since we

${ }^{2} f(u)=\Theta(g(u))$ implies that for large enough $u$, there exist positive constants, $c_{1} \leq c_{2}$, such that $c_{1} g(u) \leq f(u) \leq c_{2} g(u)$. 
need large $K$ to ensure a close approximation to $m$-weighted fairness, this also implies large queue-lengths. This can be alleviated by a virtual queue implementation of the scheduling mechanism which will be discussed in the next section.

\section{Generalizations And Implementation CONSIDERATIONS}

\section{A. Multi-hop Wireless Networks}

The model analyzed so far focuses on cellular networks with a base station to perform the scheduling, i.e., the single-hop case. The results can also be extended to cover the multihop network case. Consider a multi-hop radio network where each user is identified by a origin-destination (O-D) pair and a collection of nodes describing the route between the O-D pair. However, the scheduler will now have to be a version of the multi-hop resource allocation described in [37], [28]. The algorithm can also be extended to the case where a collection (more than one) of routes exists between an O-D pair. The congestion control algorithm in this case would be the multipath routing algorithm proposed in [17], [41], [24] along with the resource allocation algorithm in [28]. In principle, one can prove similar results as we have done in this paper for the multi-hop problem also [11], but the scheduler is not implementable in the absence of a base station since then there is no central scheduler to solve the wireless resource allocation problem. The challenging problem of obtaining reasonable distributed solutions for the scheduling problem is still the hurdle whether one considers the throughput-optimal scheduling problem as in [37], [28] or the fair scheduling problem that we have considered here.

\section{B. Reducing Delays Using Virtual Queues}

As we have discussed earlier, one penalty for achieving fairness is the possibility of large delays at the base station buffers. We can alleviate this problem by implementing the base station scheduler using virtual queues [14], [19], [22]. For each flow, the base station maintains a counter called the virtual queue. As an example, consider flow $i$. The virtual queue of flow $i$ keeps track of a virtual queue-length, where the virtual queue-length of flow $i$ is simply the length of a queue whose arrivals are the same as that of flow $i$, but whose service rate is always a fixed fraction $\rho<1$ of the actual service rate. Therefore, the size of the virtual queue (denoted by $\tilde{x}_{i}$ for flow $i$ ) will always be larger than the actual queue-length $x_{i}$. The congestion feedback given to user $i$ is $\tilde{x}_{i}[t]$ and therefore, user $i$ will reduce its arrival rate well before its real queue builds up significantly. See Figure 3 for the model from flow $i$ 's perspective.

By choosing the $\rho$ parameter appropriately the delay levels and the packet loss probabilities can be adjusted: the lower the $\rho$, the lower the actual queue-lengths. However, there is a possible loss in throughput by choosing $\rho<1$. In Section V, we will provide simulation results which show that, by choosing $\rho$ close to 1 , but not equal to 1 , we can reduce the queue-lengths dramatically while maintaining close to $100 \%$ throughput.

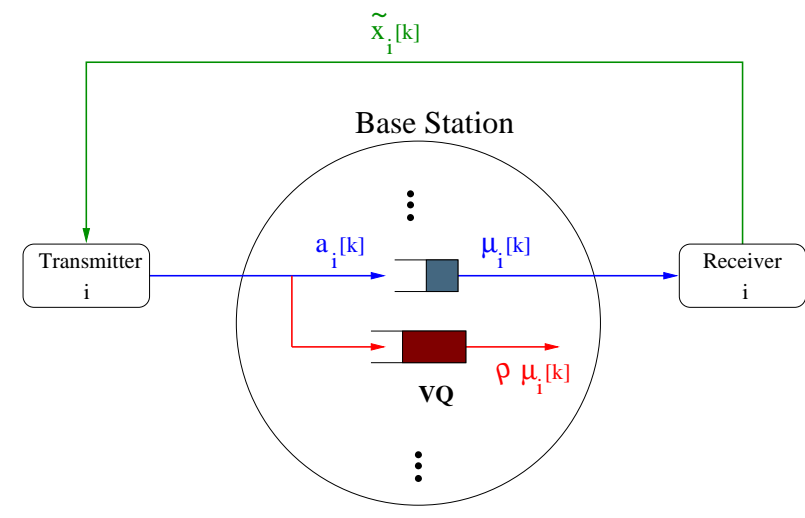

Fig. 3. The virtual queue implementation at the base station.

\section{Simulation Results}

In this section, we provide simulation results to complement the analysis in the previous sections. We present results for a ten-user scenario, and make the reasonable assumption that the channels between the base station and each of the users fade independently. The base station is only allowed to serve a single queue in a given $\operatorname{slot}^{3}$. For this scenario, the scheduling algorithm (2) is equivalent to serving the user that solves $i^{*}[t]=\underset{i \in\{1, \cdots, 10\}}{\arg \max } \eta_{i} x_{i}[t]$, at the rate $\eta_{i^{*}}[t]$. Ties are broken randomly. We let the rate vectors, $\left(\eta_{1}[t], \cdots, \eta_{10}[t]\right)$, be Poisson distributed with mean $0.4+(0.1) k$ for the $k^{t h}$ user. Also, the number of arrivals in each slot to each queue is Poisson distributed with the mean determined by the congestion controller. In the simulations, we set $\alpha_{i}=1$ for all $i$ and investigated the behavior of the queue-lengths as a function of $K$ for different $m$ values.

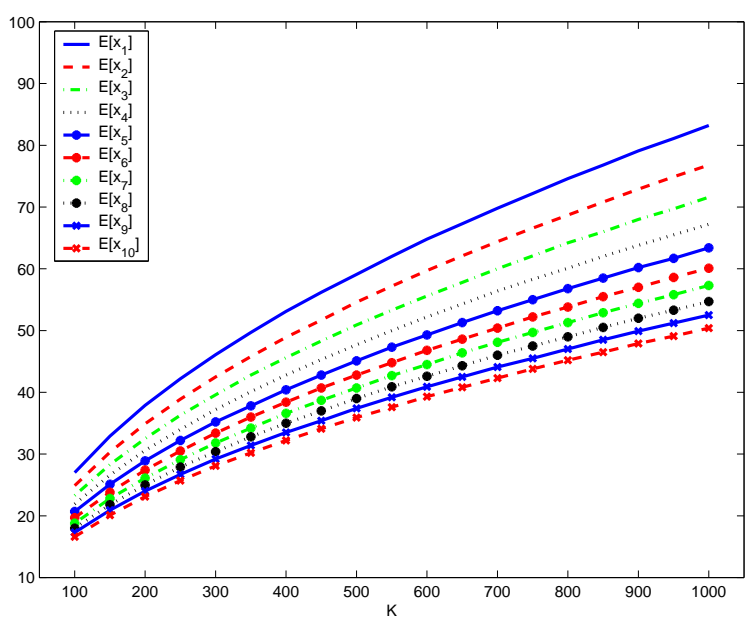

Fig. 4. Empirical change in the mean of users' queue-lengths with $K$, when $m=2$.

We start with the case of $m=2$. Figure 4 plots the average queue-length levels experienced by each flow, as a function of $K$. To confirm that the queue-lengths are proportional to $\sqrt{K}$ in accordance with our analysis, we plot the squared values

\footnotetext{
${ }^{3}$ Notice that for this model the achievable rate region for a given channel state is given by a region in the positive quadrant of $\mathbb{R}^{n}$ that lies on or below a corresponding hyperplane.
} 


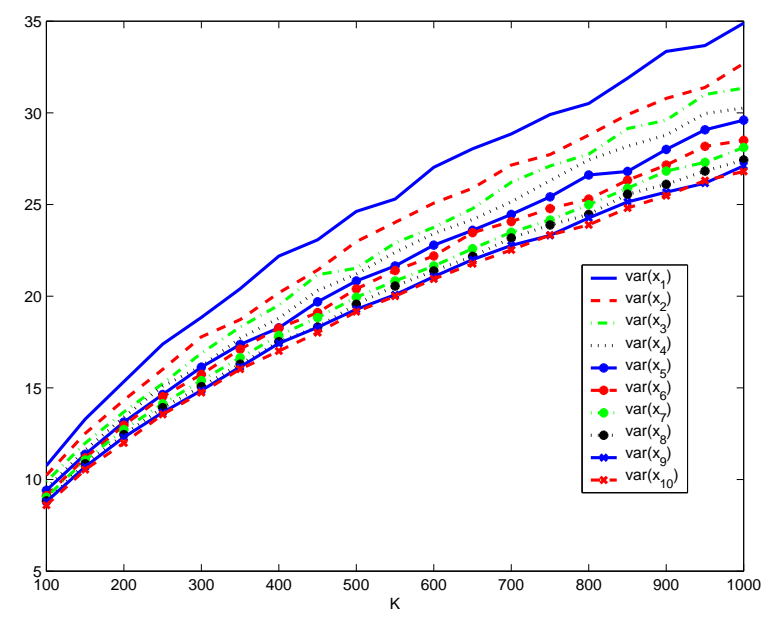

Fig. 5. Empirical variance of the queue-lengths with increasing $K$, when $m=2$.

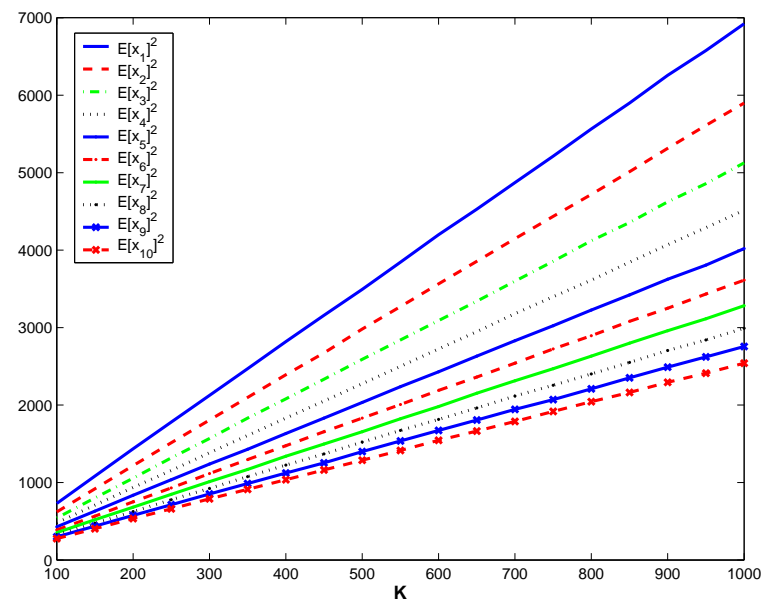

Fig. 6. Squared empirical mean values of users' queue-lengths with $K$, when $m=2$.

of the mean in Figure 6. In Figure 5, the variances of the queue-lengths are depicted and it can be seen in Figure 7 that they are also of the form $\sqrt{K}$. Therefore, the queuelengths are more and more clustered around the mean level as $K$ increases. This observation agrees with our theoretical arguments in Section III.

The average service rates provided to the flows is plotted in Figure 8 with differing $K$, when $m=2$. Clearly, $K$ does not have a significant impact on these levels. It is of interest to determine whether these rates are allocated in a fair manner. We will study this aspect later on in this section.

Next, we take $m=1$. In this case, our analysis predicts that the mean queue-length levels change linearly with $K$. Figure 9 verifies this expectation and Figure 10 plots the mean service rate levels as a function of $K$. Again, we observe that the average service rates appear to be the same for different $K$.

Finally, we consider the case when $m=0.5$. Figure 11 plots the mean queue-length levels of the queues as a function of $K$. Figure 12 depicts the change in the mean service rates with $K$. We observe once again that the mean service rates are not significantly affected by the $K$ parameter. On the other hand,

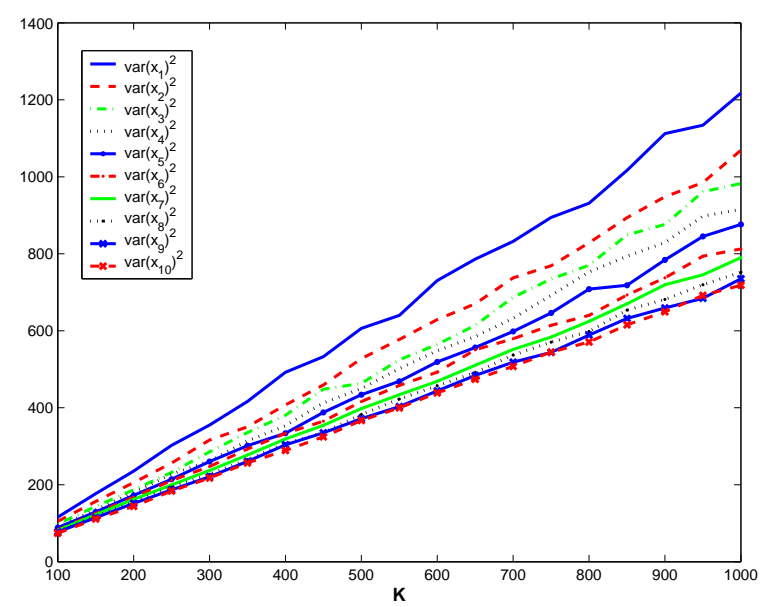

Fig. 7. Squared empirical variance of the queue-lengths with increasing $K$, when $m=2$.

the average queue-length levels increase as $K^{2}$, in agreement with our theoretical analysis.

Next, we compare the above implementation of a queuelength-based base station scheduler and an end-to-end congestion controller with the $m$-weighted fair scheduler, similarly designed with the scheduler suggested by [39]. This scheduler is described next.

$m$-Weighted FAIR SCHEdUler [39]: The scheduler keeps track of the average service rates provided to each of the flows in the last $t_{c}$ slots. We denote this parameter by $T_{i}[t]$ for the $i^{\text {th }}$ flow in slot $t$. Then, given the achievable service rates $\left\{\eta_{i}[t]\right\}$, the scheduler serves the queue, $i^{*}[t]$, that satisfies

$$
i^{*}[t]=\arg \max _{i=1,2} \frac{\eta_{i}[t]}{\left(T_{i}[t]\right)^{\frac{1}{m}}},
$$

where $T_{i}[t]$ is updated using an exponential weighted low-pass filter as follows:

$$
T_{i}[t+1]= \begin{cases}\left(1-\frac{1}{t_{c}}\right) T_{i}[t]+\frac{\eta_{i}[t]}{t_{c}} & i=i^{*}[t] \\ \left.1-\frac{1}{t_{c}}\right) T_{i}[t] & i \neq i^{*}[t]\end{cases}
$$

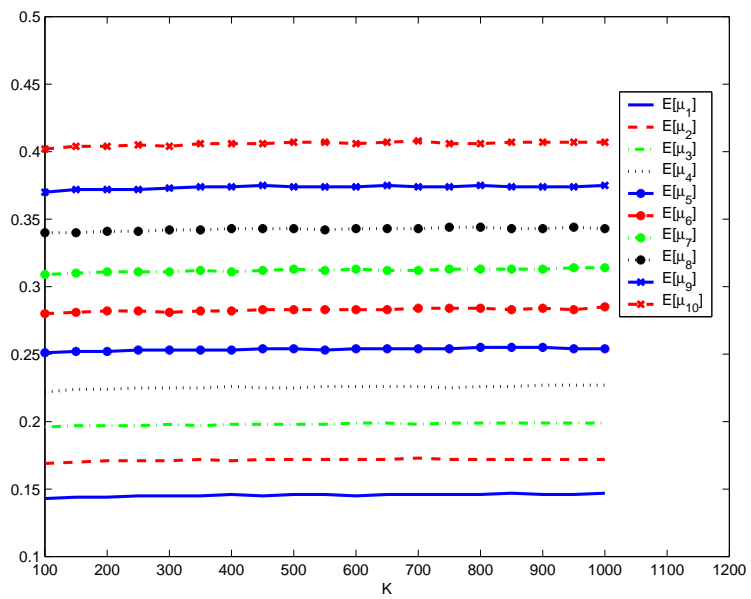

Fig. 8. Empirical average of the service rates provided to the flows for various $K$ with $m=2$. 


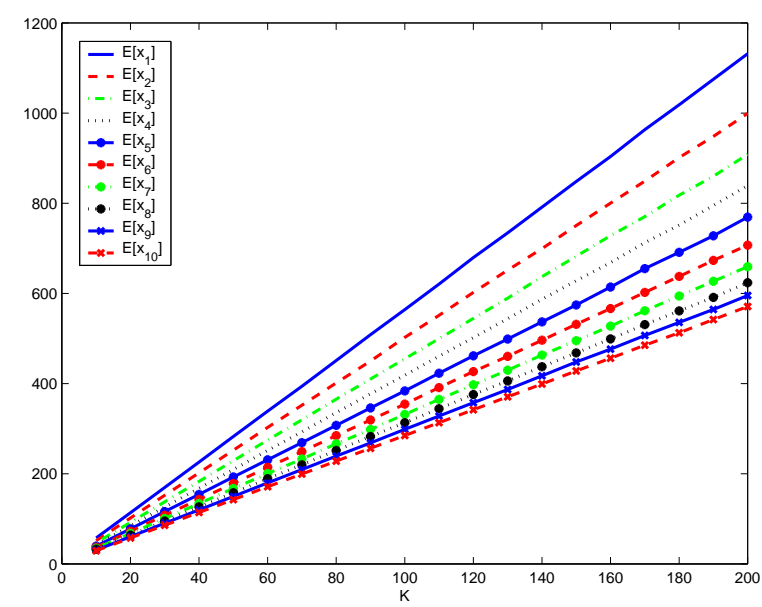

Fig. 9. Empirical change in the mean of users' queue-lengths with $K$, when $m=1$.

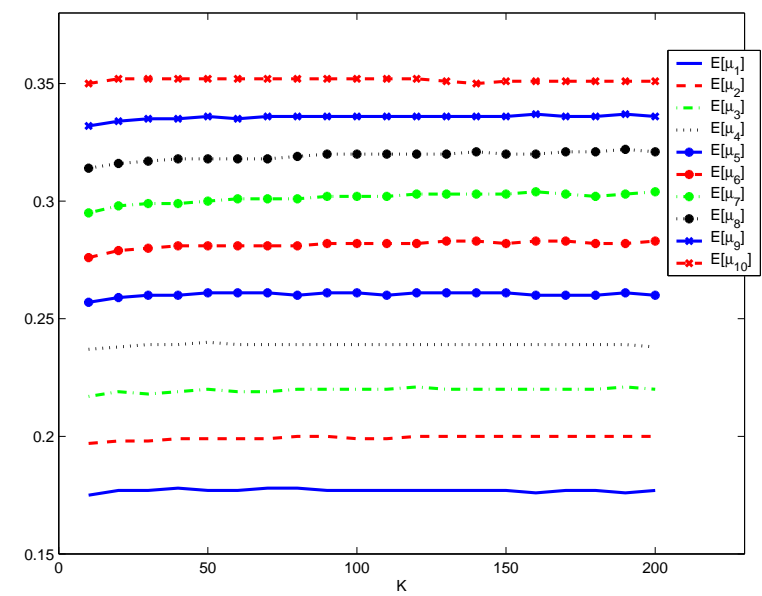

Fig. 10. Mean service rates with increasing $K$, when $m=1$.

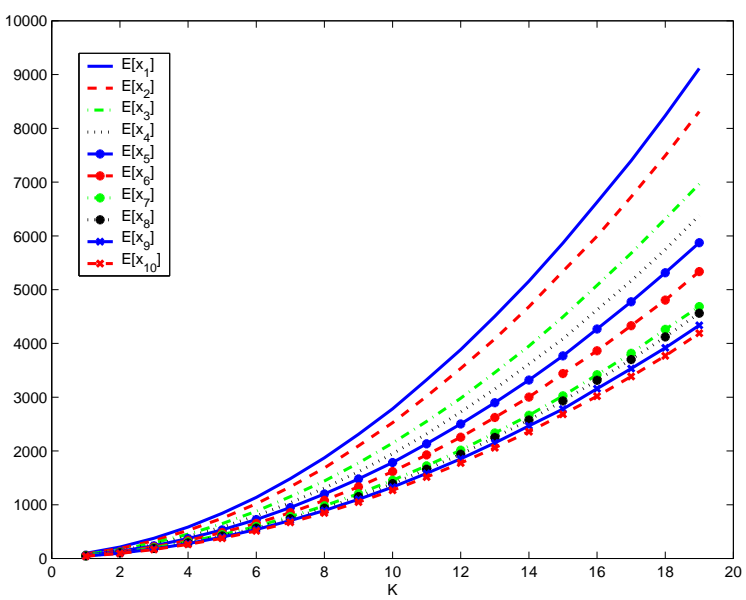

Fig. 11. Empirical change in the mean of users' queue-lengths with $K$, when $m=0.5$.

In our simulations, we let $t_{c}$ to be 100 . Notice that $T_{i}[t]$ serves as an empirical average service rate provided to flow $i$ until time $t$. Hence, this scheduler gives priority to those flows that haven't received enough service in the history.

Table 1 shows the empirical average obtained by this $m$ -

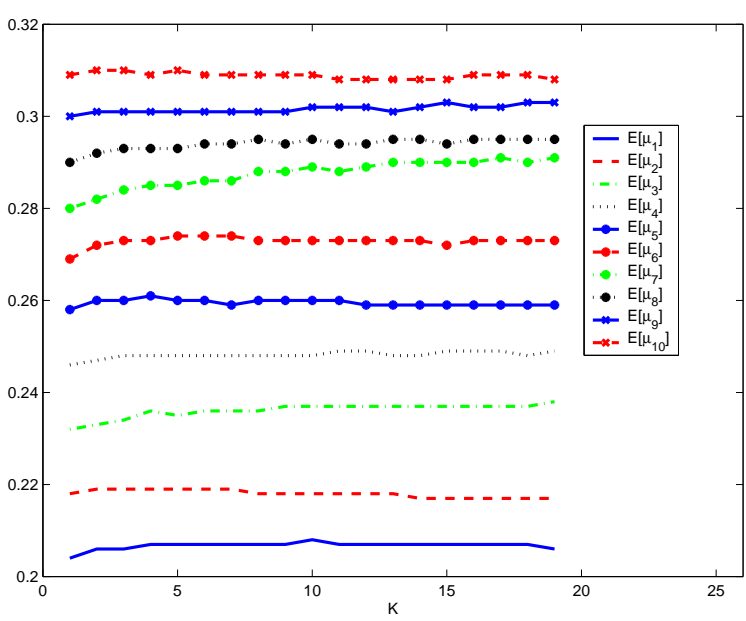

Fig. 12. Mean service rates with increasing $K$, when $m=0.5$.

\begin{tabular}{|l||c|c||c|c||c|c|}
\hline$\# \backslash(m)$ & $\mathrm{Q}(2)$ & $\mathrm{M}(2)$ & $\mathrm{Q}(1)$ & $\mathrm{M}(1)$ & $\mathrm{Q}(1 / 2)$ & $\mathrm{M}(1 / 2)$ \\
\hline \hline User 1 & 0.147 & 0.147 & 0.177 & 0.177 & 0.206 & 0.202 \\
\hline User 2 & 0.172 & 0.173 & 0.200 & 0.198 & 0.217 & 0.217 \\
\hline User 3 & 0.199 & 0.199 & 0.220 & 0.218 & 0.238 & 0.231 \\
\hline User 4 & 0.227 & 0.228 & 0.238 & 0.239 & 0.249 & 0.244 \\
\hline User 5 & 0.254 & 0.258 & 0.260 & 0.259 & 0.259 & 0.256 \\
\hline User 6 & 0.285 & 0.288 & 0.283 & 0.279 & 0.273 & 0.267 \\
\hline User 7 & 0.314 & 0.316 & 0.304 & 0.297 & 0.291 & 0.277 \\
\hline User 8 & 0.343 & 0.345 & 0.321 & 0.316 & 0.295 & 0.287 \\
\hline User 9 & 0.375 & 0.373 & 0.336 & 0.333 & 0.303 & 0.296 \\
\hline User 10 & 0.407 & 0.403 & 0.351 & 0.351 & 0.308 & 0.306 \\
\hline
\end{tabular}

TABLE I

COMPARISON OF OUR QUEUE-LENGTH-BASED ALGORITHM (DENOTED AS $Q$ IN THE TABLE) TO THE $m$-WEIGHTED FAIR SCHEDULER (DENOTED AS $M$ IN THE TABLE). MEAN RATES UNDER THE TWO ALGORITHMS ARE PROVIDED FOR $m=2,1$, AND $1 / 2$.

weighted fair scheduler along with our results for differing $m$ parameters. We observe that the empirical mean service rate allocated to the users under the $m$-weighted fair algorithm in [39] and our scheduler are in fact nearly identical as is to be expected.

Finally, we simulate the virtual queue implementation described in Section IV for the case when $m=1$ and $K=100$. We demonstrate the effect of $\rho$ on the mean queue-length levels of queues 1 and 10 in Figure 13. Here, we plot the users that experience the largest and smallest mean queuelengths in order to avoid confusion. The rest of the queues exhibit similar behaviors.

It can be seen from this simulation that modifying $\rho$ has a dramatic impact on the mean queue-length levels as we had argued. Of course, the choice of virtual queue parameter $\rho$ will also have an effect on the mean service rates as shown in Figure 14. For example, if $\rho$ is chosen to be 0.99 (which leads to a dramatic decrease in the queue-lengths as seen from Figure 13), the mean service rate for users do not decrease significantly. Thus, we see that by sacrificing throughput minimally one can dramatically reduce the queue-lengths which is consistent with the observation for the Internet [14], [19], [22]. 


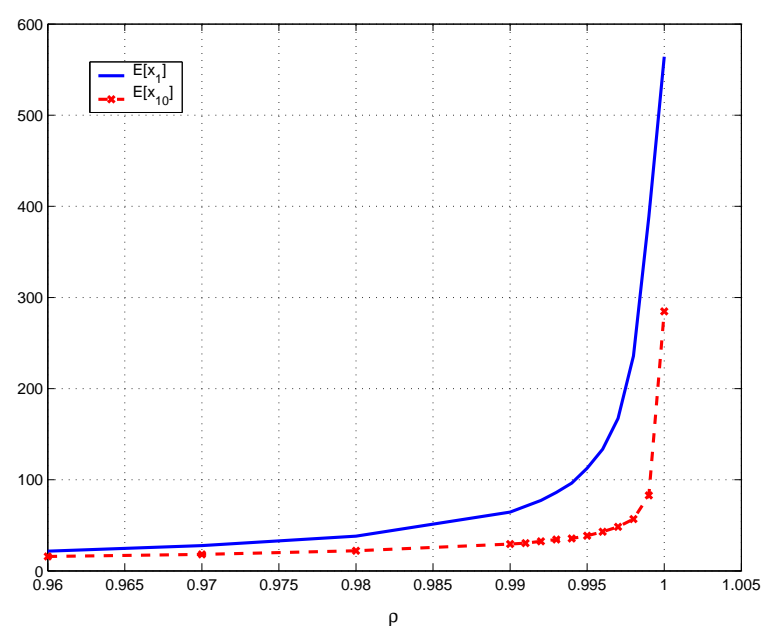

Fig. 13. Empirical change in the mean of users' queue-lengths with $\rho$, when $m=1, K=100$.

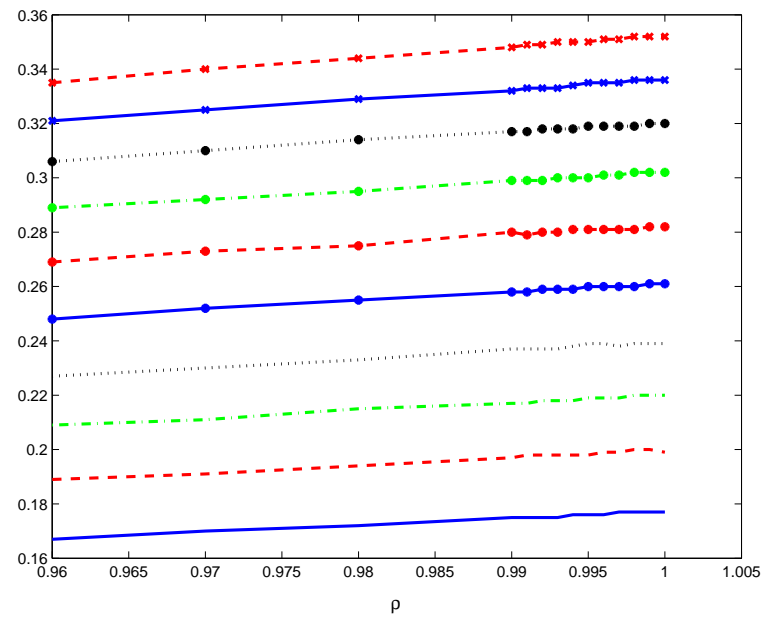

Fig. 14. Mean service rates with increasing $\rho$, when $m=1, K=100$.

\section{CONCLUSIONS}

We showed that the interaction between the end-to-end congestion controller and the local queue-length-based scheduler interestingly results in an $m$ - weighted fair allocation of the services. Furthermore, using virtual queues, the buffer levels are kept low and hence the delays experienced by the flows are also low. We also provided a characterization of the point of operation for the queue-length and service rate levels for each of the flows.

\section{APPENDIX}

Proof: [Proof of (10)] We start by noting that the superscript $(K)$ is omitted to facilitate the presentation. We write the evolution of the $i^{t h}$ queue as

$$
x_{i}[t+1]=x_{i}[t]+a_{i}[t]-\mu_{i}[t]+u_{i}[t],
$$

where $u_{i}[t]$ denotes the amount of unused service that is offered to the queue. Clearly, $u_{i}[t] \leq \mu_{i}[t] \leq \hat{\eta}$. Therefore, if $x_{i}[t]>\hat{\eta}$, then we have $u_{i}[t]=0$.

Now we study the conditional mean drift of $W(\cdot)$.

$$
\begin{aligned}
& E\left[\Delta W_{t}(\mathbf{x})\right] \\
& =\sum_{i=1}^{n} \frac{E\left[\left(x_{i}[t+1]-\stackrel{\star}{x}_{i}\right)^{2}-\left(x_{i}[t]-\stackrel{\star}{x}_{i}\right)^{2} \mid \mathbf{x}[t]=\mathbf{x}\right]}{2} \\
& =\frac{1}{2} \sum_{i=1}^{n} E\left[\left(x_{i}[t+1]-x_{i}[t]\right)\right. \\
& \left.\quad\left(x_{i}[t+1]+x_{i}[t]-2 \stackrel{\star}{x}_{i}\right) \mid \mathbf{x}[t]=\mathbf{x}\right] .
\end{aligned}
$$

By substituting (16) and dropping the time variable $t$ for convenience, we get

$$
\begin{gathered}
E\left[\Delta W_{t}(\mathbf{x})\right] \\
=\frac{1}{2} \sum_{i=1}^{n} E\left[\left(a_{i}-\mu_{i}+u_{i}\right)\left(2 x_{i}+a_{i}-\mu_{i}+u_{i}-2 \stackrel{\star}{x}_{i}\right) \mid \mathbf{x}\right] \\
\leq \sum_{i=1}^{n}\left\{\left(x_{i}-\stackrel{\star}{x}_{i}\right)\left(\min \left\{\frac{\alpha_{i} K}{\left(x_{i}\right)^{m}}, M\right\}-\bar{\mu}_{i}+E\left[u_{i} \mid \mathbf{x}\right]\right)\right. \\
\quad+\frac{E\left[a_{i}^{2}+\mu_{i}^{2}+u_{i}^{2} \mid \mathbf{x}\right]}{2}+E\left[u_{i}\left(a_{i}-\mu_{i}\right) \mid \mathbf{x}\right] \\
\left.\quad-\min \left\{\frac{\alpha_{i} K}{\left(x_{i}\right)^{m}}, M\right\} \bar{\mu}_{i}\right\},
\end{gathered}
$$

where $\bar{\mu} \in \arg \max _{\eta \in \mathcal{C}} \sum_{i=1}^{n} x_{i} \eta_{i}$, and $\mu \in \arg \max _{\eta \in \mathcal{C}_{s}} \sum_{i=1}^{n} x_{i} \eta_{i}$. Recall that $E\left[a_{i}^{2} \mid \mathbf{x}\right] \leq A$, for some finite $A$. Also, $\mu_{i}<\hat{\eta}$ implies that $E\left[u_{i}^{2}+\mu_{i}^{2} \mid \mathbf{x}\right]<2 \hat{\eta}^{2}$. Further, observing that $u_{i}$ takes positive values only if $a_{i}<\mu_{i}$ implies that $E\left[u_{i}\left(a_{i}-\right.\right.$ $\left.\left.\mu_{i}\right) \mid \mathbf{x}\right] \leq 0$. Therefore, we can upper-bound all the terms in the last line of the above expression by a finite value, say $B$, independently of $K$. Hence, we obtain

$$
\begin{aligned}
E\left[\Delta W_{t}(\mathbf{x})\right] \leq B & +\sum_{i=1}^{n}\left[\left(x_{i}-\stackrel{\star}{x}_{i}\right)\right. \\
& \left.\left(\min \left\{\frac{\alpha_{i} K}{\left(x_{i}\right)^{m}}, M\right\}-\bar{\mu}_{i}+E\left[u_{i} \mid \mathbf{x}\right]\right)\right] .
\end{aligned}
$$

For all $j$ with $x_{j}>\stackrel{\star}{x}_{j}$, we have $u_{j}=0$ when $K$ is large since we have $x_{j}>\hat{x}_{j}>\hat{\eta}$ when $K$ is taken large enough. We already argued that there cannot be any unused service if the queue-length is larger than $\hat{\eta}$. On the other hand, for all $m$ with $x_{m} \leq^{\star}{ }_{m}$, we have $E\left[u_{m} \mid \mathbf{x}\right] \geq 0$. Combining these two observations, we obtain the following upper bound:

$\left(x_{i}-\stackrel{\star}{x}_{i}\right) E\left[u_{i} \mid \mathbf{x}\right] \leq 0, \forall i$, for large K. Thus,

$E\left[\Delta W_{t}(\mathbf{x})\right]$

$$
\begin{aligned}
& \leq \sum_{i=1}^{n}\left(x_{i}-\stackrel{\star}{x}_{i}\right)\left(\min \left\{\frac{\alpha_{i} K}{\left(x_{i}\right)^{m}}, M\right\}-\bar{\mu}_{i}\right)+B \\
& =\sum_{i=1}^{n}\left(x_{i}-\stackrel{\star}{x}_{i}\right)\left(\min \left\{\frac{\alpha_{i} K}{\left(x_{i}\right)^{m}}, M\right\}-\stackrel{\star}{\mu}_{i}\right) \\
& +\sum_{i=1}^{n}\left(x_{i}-\stackrel{\star}{x}_{i}\right)\left(\stackrel{\star}{\mu}_{i}-\bar{\mu}_{i}\right)+B \\
& \leq \sum_{i=1}^{n}\left(x_{i}-\stackrel{\star}{x}_{i}\right)\left(\min \left\{\frac{\alpha_{i} K}{\left(x_{i}\right)^{m}}, M\right\}-\stackrel{\star}{\mu}_{i}\right)+B,
\end{aligned}
$$


where the last step follows from the observation in the proof of Proposition 2 that

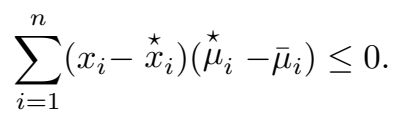

We let $i^{*}=\arg \max _{i}\left|x_{i}-\stackrel{\star}{x}_{i}\right|$ and re-write the upper bound as

$E\left[\Delta W_{t}(\mathbf{x})\right] \leq-\left|x_{i^{*}}-\stackrel{\star}{x}_{i^{*}}\right|\left|\min \left\{\frac{\alpha_{i^{*}} K}{\left(x_{i^{*}}\right)^{m}}, M\right\}-\stackrel{\star}{\mu_{i^{*}}}\right|+B$.

Now, if $\min \left\{\frac{\alpha_{i^{*} K}}{\left(x_{i^{*}}\right)^{m}}, M\right\}=M$, then

$$
\left|\min \left\{\frac{\alpha_{i^{*}} K}{\left(x_{i^{*}}\right)^{m}}, M\right\}-\stackrel{\star}{\mu}_{i^{*}}\right|=M-\stackrel{\star}{\mu}_{i^{*}}>\hat{\eta} .
$$

If $\min \left\{\frac{\alpha_{i^{*} K} K}{\left(x_{i^{*}}\right)^{m}}, M\right\}=\frac{\alpha_{i^{*} K}}{\left(x_{i^{*}}\right)^{m}}$, then

$$
\begin{aligned}
& \left|\min \left\{\frac{\alpha_{i^{*}} K}{\left(x_{i^{*}}\right)^{m}}, M\right\}-\stackrel{\star}{\mu_{i^{*}}}\right|=\stackrel{\star}{i_{i^{*}}}\left|1-\left(\frac{\stackrel{\star}{x_{i^{*}}}}{x_{i^{*}}}\right)^{m}\right|
\end{aligned}
$$

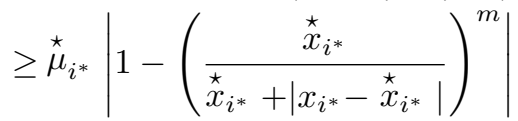

Suppose that $\|\stackrel{\star}{\mathbf{x}}-\mathbf{x}\| \geq c(K)^{\frac{1}{2 m}}$, where the choice of $c>0$ will be specified later. Noting the inequalities

$$
\left|x_{i^{*}}-\stackrel{\star}{x_{i}^{*}}\right| \leq\|\mathbf{x}-\stackrel{\star}{\mathbf{x}}\| \leq \sqrt{n}\left|x_{i^{*}}-\stackrel{\star}{x_{i}^{*}}\right|,
$$

we can write

$$
\begin{gathered}
\left|1-\left(\frac{\stackrel{\star}{x}_{i^{*}}}{\stackrel{\star}{x}_{i^{*}}+\left|x_{i^{*}}-\stackrel{\star}{x}_{i^{*}}\right|}\right)^{m}\right| \stackrel{\star}{\mu_{i^{*}}} \\
\geq \stackrel{\star}{\mu}_{i^{*}}\left|1-\frac{1}{\left(1+\frac{c\left({\stackrel{\star}{i^{*}}}^{1 / m}\right.}{\sqrt{n} \alpha_{i^{*}}^{1 / m}}(K)^{-\frac{1}{2 m}}\right)^{m}}\right| \\
\geq \stackrel{\star}{\mu}_{i^{*}}\left|1-\frac{1}{1+\psi m}\right|=\stackrel{\star}{\mu}_{i^{*}} \frac{\psi m}{1+\psi m},
\end{gathered}
$$

where $\psi:=\frac{c\left(\hat{(}_{i^{*}}\right)^{1 / m}}{\sqrt{n} \alpha_{i^{*}}^{1 / m}}(K)^{-\frac{1}{2 m}}$ and the last step follows from Taylor's expansion: $(1+\psi)^{m} \geq 1+m \psi$, for $\psi>0$. Clearly, for large enough $K$, the expression in (17) can be made smaller than $\hat{\eta}$. Therefore, for large $K$ we can write

$$
\begin{aligned}
& E\left[\Delta W_{t}(\mathbf{x})\right] \\
& \leq-\left|x_{i^{*}}-\stackrel{\star}{x}_{i^{*}}\right|\left(\stackrel{\star}{\stackrel{\star}{H}_{i^{*}}} \frac{\psi m}{1+\psi m}-\frac{B}{\left|x_{i^{*}}-\stackrel{\star}{x}_{i^{*}}\right|}\right) \\
& \leq-\left|x_{i^{*}}-\stackrel{\star}{x}_{i^{*}}\right|\left(\frac{m c\left(\stackrel{\star}{\mu}_{i^{*}}\right)^{1+\frac{1}{m}}}{\sqrt{n} \alpha_{i^{*}}^{1 / m}(K)^{\frac{1}{2 m}}+m c\left(\left(_{i^{*}}^{\star}\right)^{\frac{1}{m}}\right.}\right. \\
& \left.-\frac{B \sqrt{n}}{c(K)^{\frac{1}{2 m}}}\right) \\
& \leq-\frac{\left|x_{i^{*}}-\stackrel{\star}{x}_{i^{*}}\right|}{(K)^{\frac{1}{2 m}}}\left(\frac{m c\left(\stackrel{\star}{\mu}_{i^{*}}\right)^{1+\frac{1}{m}}}{\sqrt{n} \alpha_{i^{*}}^{1 / m}+m c\left(\stackrel{\star}{\mu}_{i^{*}}\right)^{\frac{1}{m}}}-\frac{B \sqrt{n}}{c}\right)
\end{aligned}
$$

It is not difficult to see that the expression in the parentheses can be made strictly negative by choosing $c$ sufficiently large, independent of $K$. Then the previous expression becomes $E\left[\Delta W_{t}(\mathbf{x})\right] \leq-\frac{\hat{\delta}}{(K)^{\frac{1}{2 m}}}\left|x_{i^{*}}-\stackrel{\star}{x}_{i^{*}}\right|$, for some $\hat{\delta}>0$. Using the fact that $\|\mathbf{x}-\stackrel{\star}{\mathbf{x}}\| \leq \sqrt{n}\left|x_{i^{*}}-\stackrel{\star}{x}_{i^{*}}\right|$, we can further write

$$
E\left[\Delta W_{t}(\mathbf{x})\right] \leq-\frac{\delta}{(K)^{\frac{1}{2 m}}}\|\mathbf{x}-\stackrel{\star}{\mathbf{x}}\|,
$$

for all $\|\mathbf{x}-\stackrel{\star}{\mathbf{x}}\| \geq c(K)^{\frac{1}{2 m}}$, for $K$ large enough and where $\delta:=\frac{\hat{\delta}}{\sqrt{n}}$.

When $\|\mathbf{x}-\stackrel{\star}{\mathbf{x}}\|<c(K)^{\frac{1}{2 m}}$, it is not difficult to see that $E\left[\Delta W_{t}(\mathbf{x})\right] \leq \zeta$ for some $\zeta>0$. Combining the previous two inequalities completes the proof.

\section{REFERENCES}

[1] R. Agrawal, A. Bedekar, R. J. La, and V. Subramanian. Class and channel condition based weighted proportionally fair scheduler. In Proceedings of the International teletraffic Congress, pages 553-65, 2001.

[2] T. Alpcan and T. Başar. A utility-based congestion control scheme for internet-style networks with delay. In Proceedings of IEEE Infocom, San Francisco, California, March-April 2003.

[3] M. Andrews, K. Kumaran, K.Ramanan, A. Stolyar, R. Vijayakumar, and P. Whiting. Providing quality of service over a shared wireless link. IEEE Communications Magazine, February 2001.

[4] M. Armony and N. Bambos. Queueing dynamics and maximal throughput scheduling in switched processing systems. Technical Report Netlab2001-09/01, Stanford University.

[5] S. Asmussen. Applied Probability and Queues. Springer-Verlag, New York, 2003.

[6] D. Bertsekas. Nonlinear Programming. Athena Scientific, Belmont, MA, 1995.

[7] D. Bertsekas and R. Gallager. Data Networks. Prentice Hall, Englewood Cliffs, NJ, 1987.

[8] S. C. Borst. User-level performance of channel-aware scheduling algorithms in wireless data networks. In Proceedings of IEEE INFOCOM, 2003.

[9] S.C. Borst and P.A. Whiting. Dynamic rate control algorithms for HDR throughput optimization. In Proceedings of IEEE INFOCOM, pages 976-985, 2001.

[10] R. Buche and H. J. Kushner. Control of mobile communication systems with time-varying channels via stability methods. IEEE Transactions on Automatic Control, 2004. To appear.

[11] A. Eryilmaz. Efficient and fair scheduling for wireless networks. PhD thesis, University of Illinois at Urbana, Champaign, August 2005.

[12] A. Eryilmaz, R. Srikant, and J. Perkins. Stable scheduling policies for fading wireless channels. In Proceedings of IEEE International Symposium on Information Theory, 2003. To appear in the IEEE/ACM Transactions on Networking, February, 2005.

[13] A. Eryilmaz, R. Srikant, and J. R. Perkins. Stable scheduling policies for fading wireless channels. IEEE/ACM Transactions on Networking, 13:411-425, April 2005.

[14] R. J. Gibbens and F. P. Kelly. Resource pricing and the evolution of congestion control. Automatica, 35:1969-1985, 1999.

[15] A. Jalali, R. Padavoni, and R. Pankaj. Data throughput of CDMA-HDR: a high efficiency-high data rate personal communication system. In Proceedings IEEE Vehicular Technology Conference, pages 1854-1858.

[16] F. P. Kelly. Charging and rate control for elastic traffic. European Transactions on Telecommunications, 8:33-37, 1997.

[17] F. P. Kelly, A. Maulloo, and D. Tan. Rate control in communication networks: shadow prices, proportional fairness and stability. Journal of the Operational Research Society, 49:237-252, 1998.

[18] H. Khalil. Nonlinear Systems. 2nd edition, Prentice Hall, Upper Saddle River, NJ, 1996.

[19] S. Kunniyur and R. Srikant. Analysis and design of an adaptive virtual queue algorithm for active queue management. In Proceedings of ACM Sigcomm, pages 123-134, San Diego, CA, August 2001.

[20] S. Kunniyur and R. Srikant. A time-scale decomposition approach to adaptive ECN marking. IEEE Transactions on Automatic Control, 47(6):882-894, June 2002.

[21] H. J. Kushner and P. A. Whiting. Convergence of proportional-fair sharing algorithms under general conditions, February 2003. Preprint. 
[22] A. Lakshmikantha, C. Beck, and R. Srikant. Robustness of real and virtual queue based active queue management schemes. IEEE/ACM Transactions on Networking, 2004. To appear. An earlier version appeared in the Proceedings of the American Control Conference, June 2003.

[23] R. Leelahakriengkrai and R. Agrawal. Scheduling in multimedia wireless networks. In Proceedings of ITC, Brazil, 2001.

[24] X. Lin and N. Shroff. The multi-path utility maximization problem. In Proceedings of the Allerton Conference on Communications, Control and Computing, Monticello, IL, October 2003.

[25] X. Liu, E. Chong, and N. Shroff. Opportunistic transmission scheduling with resource-sharing constraints in wireless networks. IEEE Journal on Selected Areas in Communications, 19(10):2053-2064, October 2001.

[26] Y. Liu and E. Knightly. Opportunistic fair scheduling over multiple wireless channels. In Proceedings of IEEE INFOCOM, San Francisco, CA, April 2003.

[27] S. H. Low and D. E. Lapsley. Optimization flow control, I: Basic algorithm and convergence. IEEE/ACM Transactions on Networking, pages 861-875, December 1999.

[28] M.J. Neely, E. Modiano, and C.E. Rohrs. Dynamic power allocation and routing for time varying wireless networks. Proceedings of IEEE INFOCOM, April 2003.

[29] J. R. Norris. Markov Chains. Cambridge University Press, Cambridge, United Kingdom, 1997.

[30] F. Paganini. A global stability result in network flow control. Systems and Control Letters, 46(3):153-163, 2002.

[31] A. G. Pakes. Some conditions on the ergodicity and recurrence of Markov chains. Operations Research, 17, 1969.

[32] S. Shakkottai, R. Srikant, and A. Stolyar. Pathwise optimality of the exponential scheduling rule for wireless channels. Advances in Applied Probability, 36:1021-1045, 2004.

[33] S. Shakkottai and A. Stolyar. Scheduling for multiple flows sharing a time-varying channel: The exponential rule. Translations of the AMS, Series 2, A volume in memory of F. Karpelevich, 207, 2002.

[34] R. Srikant. The Mathematics of Internet Congestion Control. Birkhauser 2004.

[35] A. Stolyar. Maximizing queueing network utility subject to stability: Greedy primal-dual algorithm. Queueing Systems, 50(4):401-457, 2005

[36] V. Subramanian and R. Agrawal. A stochastic approximation analysis of channel condition aware wireless scheduling algorithms. In Proceedings of the INFORMS Telecommunications Conference, 2002.

[37] L. Tassiulas. Scheduling and performance limits of networks with constantly varying topology. IEEE Transactions on Information Theory, pages 1067-1073, May 1997.

[38] L. Tassiulas and A. Ephremides. Stability properties of constrained queueing systems and scheduling policies for maximum throughput in multihop radio networks. IEEE Transactions on Automatic Control, pages 1936-1948, December 1992.

[39] D. N. Tse. Multi-user diversity and proportional fairness. US Patent 6449490.

[40] P. Viswanath, D. Tse, and R. Laroia. Opportunistic beamforming using dumb antennas. IEEE Transactions on Information Theory, 48(6):12771294, June 2002.

[41] W.-H. Wang, M. Palaniswami, and S. H. Low. Optimal flow control and routing in multi-path networks. Performance Evaluation, pages 119-132, 2003.

[42] J.T. Wen and M. Arcak. A unifying passivity framework for network flow control. In Proceedings of IEEE INFOCOM, April 2003.

[43] H. Yaiche, R. R. Mazumdar, and C. Rosenberg. A game-theoretic framework for bandwidth allocation and pricing in broadband networks. IEEE/ACM Transactions on Networking, 8(5):667-678, October 2000.

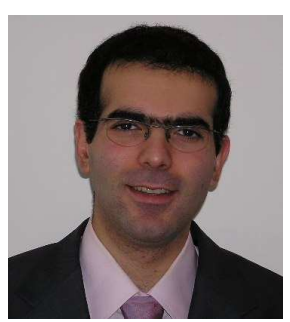

Atilla Eryilmaz (S '00-M '06) received his B.S. degree in Electrical and Electronics Engineering from Bogaziçi University, Istanbul, in 1999, and the M.S. and Ph.D. degrees in Electrical and Computer Engineering from the University of Illinois at UrbanaChampaign in 2001 and 2005, respectively. He is currently working as a Postdoctoral Associate at the Laboratory for Information and Decision Systems at the Massachusetts Institute of Technology.

His research interests include communication networks, optimal control of stochastic networks, optimization theory, stochastic processes and network coding.

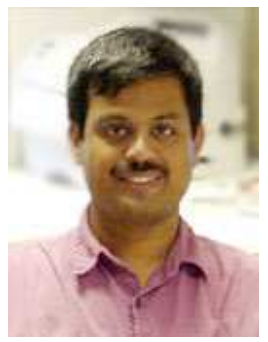

R. Srikant (S '90-M '91-SM '01-F' '06) received his B.Tech. from the Indian Institute of Technology, Madras in 1985, his M.S. and Ph.D. from the University of Illinois in 1988 and 1991, respectively, all in Electrical Engineering. He was a Member of Technical Staff at AT\&T Bell Laboratories from 1991 to 1995. He is currently with the University of Illinois at Urbana-Champaign, where he is a Professor in the Department of Electrical and Computer Engineering, and a Research Professor in the Coordinated Science Laboratory.

He was an associate editor of Automatica and the IEEE Transactions on Automatic Control, and is currently an associate editor of the IEEE/ACM Transactions on Networking. He has also served on the editorial boards of special issues of the IEEE Journal on Selected Areas in Communications and IEEE Transactions on Information Theory. He was the chair of the 2002 IEEE Computer Communications Workshop in Santa Fe, NM and is a program cochair of IEEE INFOCOM, 2007. His research interests include communication networks, stochastic processes, queueing theory, information theory, and game theory. 